Swarthmore College

Works

9-14-2015

\title{
Liquid Crystal Janus Emulsion Droplets: Preparation, Tumbling, And Swimming
}

J. Jeong
A. Gross
W.-S. Wei
F. Tu
D. Lee

See next page for additional authors

Follow this and additional works at: https://works.swarthmore.edu/fac-physics

Part of the Physics Commons

Let us know how access to these works benefits you

\section{Recommended Citation}

J. Jeong, A. Gross, W.-S. Wei, F. Tu, D. Lee, Peter J. Collings, and A. G. Yodh. (2015). "Liquid Crystal Janus Emulsion Droplets: Preparation, Tumbling, And Swimming". Soft Matter. Volume 34, 6747-6754. DOI:

10.1039/C5SM01053E

https://works.swarthmore.edu/fac-physics/240

This work is brought to you for free by Swarthmore College Libraries' Works. It has been accepted for inclusion in Physics \& Astronomy Faculty Works by an authorized administrator of Works. For more information, please contact myworks@swarthmore.edu. 
Authors

J. Jeong, A. Gross, W.-S. Wei, F. Tu, D. Lee, Peter J. Collings, and A. G. Yodh 


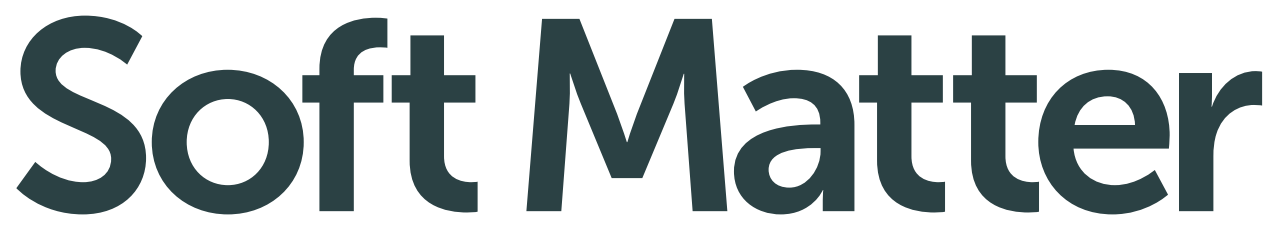

\section{www.softmatter.org}
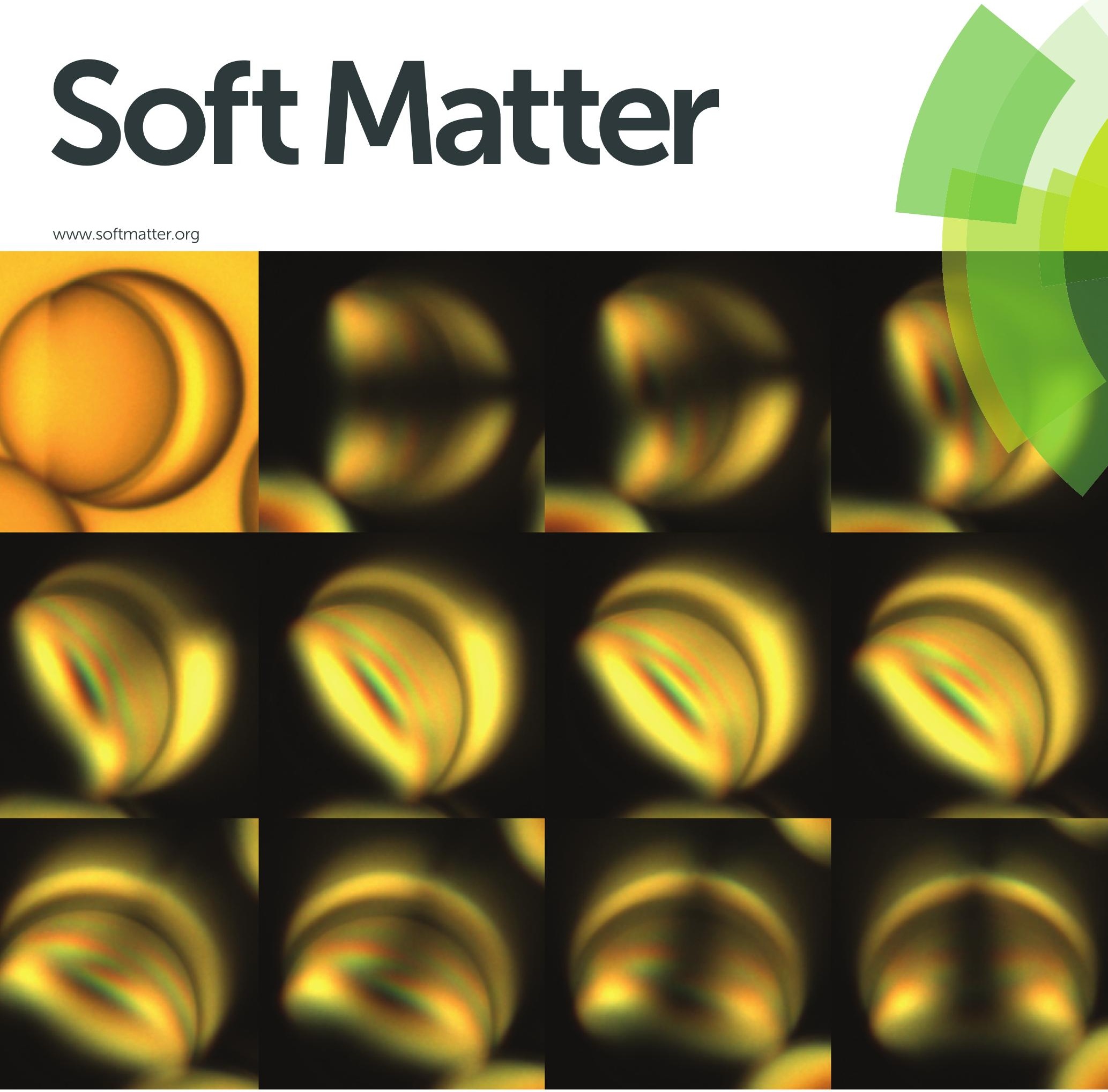

ISSN 1744-683X

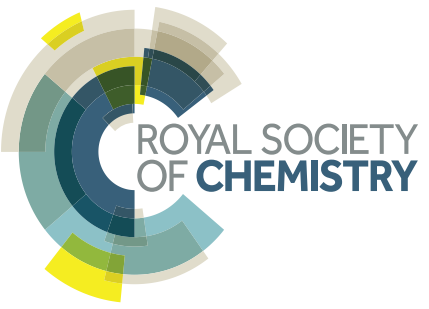

\section{PAPER}

Joonwoo Jeong et al.

Liquid crystal Janus emulsion droplets: preparation, tumbling, and swimming

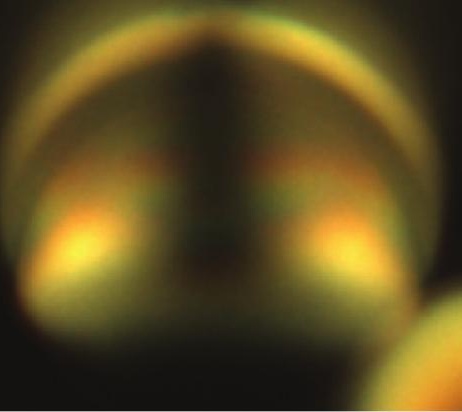




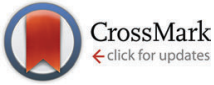

Cite this: Soft Matter, 2015 11,6747

Received 3rd May 2015 Accepted 30th June 2015 DOI: $10.1039 / \mathrm{c} 5 \mathrm{sm} 01053 \mathrm{e}$ www.rsc.org/softmatter

\title{
Liquid crystal Janus emulsion droplets: preparation, tumbling, and swimming $\dagger$
}

\author{
Joonwoo Jeong, ${ }^{\star a b}$ Adam Gross, ${ }^{c}$ Wei-Shao Wei, ${ }^{c}$ Fuquan Tu, ${ }^{d}$ Daeyeon Lee, ${ }^{d}$ \\ Peter J. Collings ${ }^{\mathrm{ce}}$ and A. G. Yodh
}

\begin{abstract}
This study introduces liquid crystal (LC) Janus droplets. We describe a process for the preparation of these droplets, which consist of nematic LC and polymer compartments. The process employs solvent-induced phase separation in emulsion droplets generated by microfluidics. The droplet morphology was systematically investigated and demonstrated to be sensitive to the surfactant concentration in the background phase, the compartment volume ratio, and the possible coalescence of multiple Janus droplets. Interestingly, the combination of a polymer and an anisotropic LC introduces new functionalities into Janus droplets, and these properties lead to unusual dynamical behaviors. The different densities and solubilities of the two compartments produce gravity-induced alignment, tumbling, and directional self-propelled motion of Janus droplets. LC Janus droplets with remarkable optical properties and dynamical behaviors thus offer new avenues for applications of Janus colloids and active soft matter.
\end{abstract}

\section{Introduction}

Janus colloids are composed of two-faced particles or droplets, of which the spatial anisotropy arises from arrangements of patches on the particle/droplet surface or from the placement of compartment volumes with distinct characteristics within the particle/droplet volume. ${ }^{1,2}$ These spatial arrangements in turn utilize variations in surface chemistry, electric/magnetic susceptibility and shape to produce anisotropic material properties on macroscopic scales. Interest in Janus colloids has grown rapidly because these systems are useful for model studies of self-assembly and active matter ${ }^{3-15}$ and are valuable for technologies such as emulsion stabilization ${ }^{16}$ and electro/ magnetophoretic displays. ${ }^{17,18}$ To date, a variety of physicochemical methods have been developed to incorporate multiple materials into single colloidal objects. For example, stamp coating, ${ }^{19}$ metal evaporation, ${ }^{13,20,21}$ seeded polymerization of monomers, ${ }^{14,22-24}$ and "Pickering emulsion" techniques ${ }^{25,26}$

\footnotetext{
${ }^{a}$ School of Natural Science, Department of Physics, Ulsan National Institute of Science and Technology (UNIST), Ulsan 689-798, Republic of Korea.

E-mail: jjeong@unist.ac.kr

${ }^{b}$ Center for Soft and Living Matter, Institute for Basic Science (IBS), Ulsan 689-798, Republic of Korea

${ }^{c}$ Department of Physics and Astronomy, University of Pennsylvania, Philadelphia, PA 19104, USA

${ }^{d}$ Department of Chemical and Biomolecular Engineering, University of Pennsylvania, Philadelphia, PA 19104, USA

${ }^{e}$ Department of Physics and Astronomy, Swarthmore College, Swarthmore, PA 19081, USA

$\dagger$ Electronic supplementary information (ESI) available. See DOI: 10.1039/ c5sm01053e
}

have been utilized to decorate regions of colloidal particles with different materials; microfluidics and electrified jetting have also been employed to create droplets and particles with multiple compartments. ${ }^{17,27-29}$ Finally, phase separation of two or more immiscible materials has been explored for the fabrication of Janus colloids, for example, using polymer blends and/or inorganic nanoparticles..$^{2,15,16,30-35}$ Surprisingly, most Janus colloids that have been synthesized to date have utilized only isotropic materials. Herein, we describe the preparation of novel liquid crystal (LC) Janus droplets by combining microfluidics and phase separation techniques, and we demonstrate how an anisotropic LC component can introduce new functionalities into Janus droplets and how these properties lead to other unusual behaviors. To our knowledge, these are the first reported LC Janus droplets.

In our LC Janus droplets, one compartment is composed of a nematic LC with temperature-dependent phase behavior and the other compartment is composed of a polymer that is immiscible with the LC. In particular, we employ $4^{\prime}$-pentyl-4biphenylcarbonitrile (5CB) as the nematic LC and poly(dimethylsiloxane) (PDMS) as the polymer. This unique combination of a LC and a polymer in a Janus droplet exhibits three different anisotropic characteristics that have not been previously explored in Janus colloids. Lastly, more complex LC droplets with two or more surrounding polymer patches/compartments were made by coalescence of droplets.

LC Janus droplets are optically inhomogeneous with respect to indices of refraction and birefringence. The nematic LC compartment of a Janus droplet is optically birefringent as a result of its aligned structure, whereas the polymer compartment 
is optically isotropic. In addition, the aligned structure inside the LC compartment can be controlled by varying the compartment geometry, anchoring conditions of the LC at droplet surfaces and interfaces, and temperature. ${ }^{36,37}$ These inhomogeneities and anisotropies in turn provide Janus colloids with potential applications in smart inks ${ }^{17,18}$ and optical manipulation. ${ }^{36,38}$

In a different context, differences in density between the LC, polymer, and aqueous background phases produce aligning and tumbling behavior. In the present case, the density of the polymer is lower than the density of the aqueous background phase, whereas the LC is denser than the background phase. An imbalance of gravitational forces induces alignment of Janus droplets and tumbling motion when the droplets are flipped. Except in the case of sedimentation, gravity is a ubiquitous but seldom used external method for inducing colloidal motion. Herein, it can be exploited to make unique structures with these Janus particles, especially at bulk fluid interfaces.

Finally, the two compartments have different solubilities in the continuous phase when dispersed in a concentrated surfactant solution. This difference in solubility induces directional self-propelled motion of the Janus droplets. The LC compartment with higher solubility is active; namely, it drives the swimming motion while the attached (inactive) polymer compartment follows. Such self-propelled LC Janus droplets offer a fascinating model system for the investigation of dynamic self-assembly and active soft matter ${ }^{11,12,39}$ in external fields with controllable hydrodynamics due to particle shape (from spheres to snowmen).

\section{Experimental section}

\section{Preparation of LC Janus droplets}

4'-Pentyl-4-biphenylcarbonitrile (5CB, purity >99.8\%) was purchased from Kingston Chemicals Ltd. Poly(dimethylsiloxane) (silicone oil, viscosity $10000 \mathrm{cSt}$ at $25{ }^{\circ} \mathrm{C}$ ), sodium dodecyl sulphate (SDS, purity >99\%), poly(vinyl alcohol) (PVA, 87\%$89 \%$ hydrolyzed and average $\left.M_{\mathrm{w}} 13000-23000\right)$, and tetradecyltrimethylammonium bromide $\left(\mathrm{C}_{14} \mathrm{TAB}\right.$, purity $\left.>99 \%\right)$ were purchased from Sigma-Aldrich. All chemicals were used as received. We dissolved the liquid crystal (5CB) and silicone oil in chloroform to make a homogeneous solution with a total concentration around $5 \%(\mathrm{wt} / \mathrm{vol})$. The mass ratios between the liquid crystal and silicone oil that were used were $1: 3,1: 1$, and $3: 1$. Because the densities of $5 \mathrm{CB}$ and silicone oil are similar (1.01 $\mathrm{g} \mathrm{cm}^{-3}$ and $0.97 \mathrm{~g} \mathrm{~cm}^{-3}$, respectively), all mass ratios can be approximated as volume ratios. A surfactant (SDS or PVA) was dissolved in deionized water $(18.2 \mathrm{M} \Omega \mathrm{cm})$ to make solutions with concentrations ranging from $0.05 \%$ to $5 \%$ (wt/vol). The other surfactant, $\mathrm{C}_{14} \mathrm{TAB}$, was also dissolved in deionized water at a concentration from $10 \%$ to $20 \%$ (wt/vol).

In brief, we dispersed the liquid crystal/polymer (5CB/silicone oil) $5 \%$ (wt/vol) solution as spherical droplets into an aqueous surfactant (PVA or SDS) $0.1 \%$ (wt/vol) solution using capillary microfluidics. ${ }^{40}$ The volume fraction of the liquid crystal/polymer solution in the surfactant solution was approximately $10 \%$ and the initial diameters of the droplets ranged from $50 \mu \mathrm{m}$ to $200 \mu \mathrm{m}$ according to the flow rates and dimensions of the microfluidics setup. The droplets were collected into uncovered borosilicate glass Petri dishes or microscope slides with wells (cavity microscope slides, Ted Pella). We stored the uncovered dishes and microscope slides in a custom-built chamber at $100 \%$ relative humidity for 6 hours, which expedited evaporation of chloroform from the droplets and minimized evaporation of water from the surfactant solution.

After the complete evaporation of chloroform, which led to phase separation of the polymer and the birefringent liquid crystals, we transferred the droplets to rectangular capillaries (0.4 $\mathrm{mm}$ height and $4 \mathrm{~mm}$ width, VitroCom) for observation. While transferring the droplets, we mixed a certain volume of the droplet solution with a surfactant solution (PVA or SDS) of known volume and concentration. Assuming that the evaporation of water from the droplet solution in the chamber with $100 \%$ relative humidity was negligible, we calculated the final concentration of the surfactant that the droplets experienced in the capillary. Then, the capillaries were sealed with silicone vacuum grease (High Vacuum Grease, Dow Corning) and stored at room temperature for 24 hours to guarantee equilibration of the Janus droplets with the new surfactant solution. In the case of the $\mathrm{C}_{14} \mathrm{TAB}$ surfactant solution, Janus droplets were observed as soon as the droplet solution was gently added to the $\mathrm{C}_{14} \mathrm{TAB}$ solution; this addition was performed on a cavity microscope slide with a single well (cavity microscope slides, Ted Pella) and the well was covered with a cover slip for observation. The final concentration of $\mathrm{C}_{14} \mathrm{TAB}$ was approximately $10 \%$ (wt/vol).

\section{Optical microscopy of LC Janus droplets}

We collected bright-field and polarized optical microscopy (POM) images with a Leica DM IRB inverted microscope using a $63 \mathrm{X}$ dry objective with cover slip thickness correction and $\mathrm{NA}=0.7$. We also used a $10 \mathrm{X}$ dry objective with $\mathrm{NA}=0.25$ for a larger field of view. The samples were rotated on a circular stage that was located between a polarizer and an analyzer. To record how the droplets tumble because of gravity, the rectangular capillaries containing the Janus droplets were flipped manually on the microscope stage. Images were taken with a color CCD camera (UC-1800DS-CL, UNIQ Vision, Inc.) under polychromatic illumination obtained from a halogen lamp. To take side-view images of Janus droplets that were aligned by gravity, we turned a Bausch and Lomb monocular microscope on its side, which was equipped with a $43 \mathrm{X}$ dry objective, a color CMOS camera (1401KEM PupilCam, Ken-A-Vision), and a halogen lamp. The monocular microscope was small and portable and could easily be turned on its side. All observations were carried out at an ambient temperature of $24^{\circ} \mathrm{C}$.

\section{Analysis of the morphologies of LC Janus droplets}

The side-view images of Janus droplets that were aligned by gravity were analyzed by the ImageJ software (National Institutes of Health). Each interface between the liquid crystal, polymer, and aqueous surfactant regions was fitted to a circle; the circle is the best fit for a set of three points on the interface, 
including two intersection points of all three interfaces. See Fig. 2a-e and the representative circle fits in Fig. S2 of the ESI. $\dagger$ If the two intersection points were not clear or if there was a visually recognizable gap (approximately 1\% of the diameter) between the best-fit circle and the corresponding interface then we excluded the droplet from the analysis; image distortion near the boundary of the field of view or Janus droplets that are not fully aligned can produce these deviations. Exceptionally, in the case of the interface between the liquid crystal and the polymer, a large gap was tolerated, considering optical artifacts that originate from birefringence of the liquid crystal and curved boundaries of the droplet. This scenario can arise because the LC-polymer interface can often be observed only through the LC compartment. From the radii, centers, and two intersection points of the three best-fit circles, we calculated the volume of each compartment and the contact angles of the interfaces at the three-phase boundary. ${ }^{35}$

\section{Results and discussions}

\section{Preparation and properties of LC Janus droplets}

We first describe the phase separation scheme for making LC Janus droplets. Fig. 1 and 2 show examples of such droplets. A capillary microfluidic device generated uniformly sized droplets, which were in turn stabilized by surfactants in the aqueous continuous phase. ${ }^{40}$ Initially, these droplets (Fig. 1a) contained a homogeneous LC/polymer solution; the droplets were dispersed in an aqueous continuous phase. Then, the organic solvent in the droplet, e.g., chloroform, started to evaporate. After complete evaporation (Fig. 1b), Janus droplets with two phase-separated compartments formed. Phase separation occurred because 5CB and high-molecular-weight PDMS are immiscible, both with each other ${ }^{41}$ and in the absence of a co-solvent, with water. The polarized optical microscopy (POM) images shown in Fig. 1c and d distinguish the polymer and LC compartments. When viewed between crossed polarizers, the optically isotropic polymer appears dark and the birefringent LC appears bright. Fig. 1e shows a sketch of each compartment and interface.

The patterns of brightness within the LC compartment result from the director configuration of the LC with respect to the directions of the crossed polarizers. The director configuration is determined by the balance of the bulk elasticity and surface anchoring of the confined LC. ${ }^{42,43}$ For example, as shown in Fig. 1e, perpendicular anchoring at the 5CB-PDMS interface and tangential anchoring at the 5CB-water (with PVA) interface cause the nematic director field (indicated by the green rods) to reconfigure. The dark band along the $z$-axis at the center of Fig. 1c arises because the LC director field along the beam path lies in the $x-z$ plane, which is perpendicular to the pass axis of the analyzer. In addition, a single point defect near the south pole of the droplet in Fig. 1e is shown in Fig. 1g (viewing direction parallel to the $z$-axis) as the central point of a Maltese cross, which suggests a radial alignment of LC directors around the defect. This topological defect in the aqueous (a)

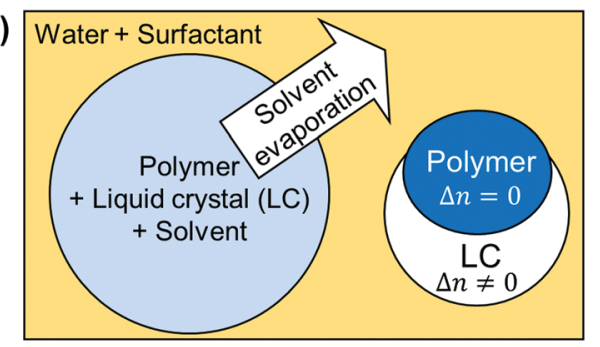

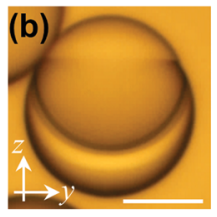

(e)

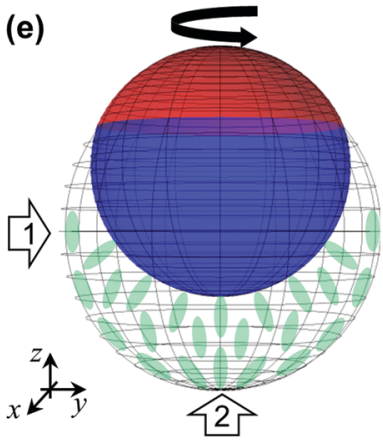

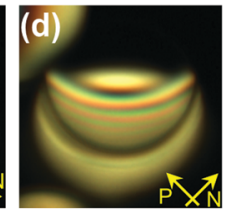

(f)

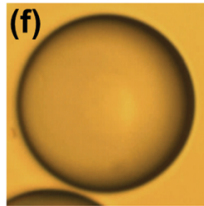

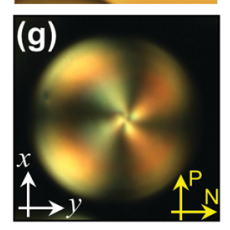

Fig. 1 Preparation and observation of Janus droplets. (a) Schematic of how evaporation of an organic solvent from a homogeneous droplet (left), consisting of a polymer and a liquid crystal (LC) in solution, leads to the formation of a Janus droplet (right) with two phase-separated compartments: polymer (blue) and LC (white). (b) Bright-field microscopy image of a Janus droplet. The polymer (upper) and LC (lower) compartments of this Janus droplet are PDMS and 5CB, respectively. The background phase is water with PVA $0.1 \%$ (wt/vol). Scale bar: $20 \mu \mathrm{m}$. Arrows $x, y$, and $z$ define our Cartesian coordinate system. (c) and (d) Polarized optical microscopy (POM) images of a Janus droplet between crossed polarizers; the directions of the pass axes of the polarizer $(\mathrm{P})$ and analyzer $(\mathrm{N})$ are shown as single-headed yellow arrows. (e) Schematic of a Janus droplet with three interfaces: water-polymer (red), polymer-LC (blue), and LC-water (transparent). Green rods represent LC mesogens with directors parallel to the LC-water interface but perpendicular to the polymer-LC interface. Note that parallel anchoring of the LC directors at the LC-water interface is controlled by PVA dissolved in the water. The rotating black arrow indicates the rotational symmetry of the droplet. The viewing directions for (b)-(d) and (f) $-(\mathrm{g})$ are shown as Arrow 1 (perpendicular to the $z$-axis) and Arrow 2 (parallel to the $z$-axis), respectively. (f) and (g) Bright-field and POM images of a Janus droplet from a viewing direction corresponding to Arrow 2 in (e).

PVA solution is a surface point defect, e.g., a boojum; it is readily observed in pre-radial configurations of a spherical LC droplet with hybrid anchoring conditions. ${ }^{37}$ In contrast, in an aqueous SDS background solution, a hedgehog point defect forms in the LC compartment; this type of defect is typically observed at the center of spherical LC droplets with uniform homeotropic anchoring.

The morphology of LC Janus droplets can be controlled by the type and concentration of the surfactants, as well as the volume ratio of the two phase-separated compartments. Fig. 2a illustrates the relationship between the morphology of a 

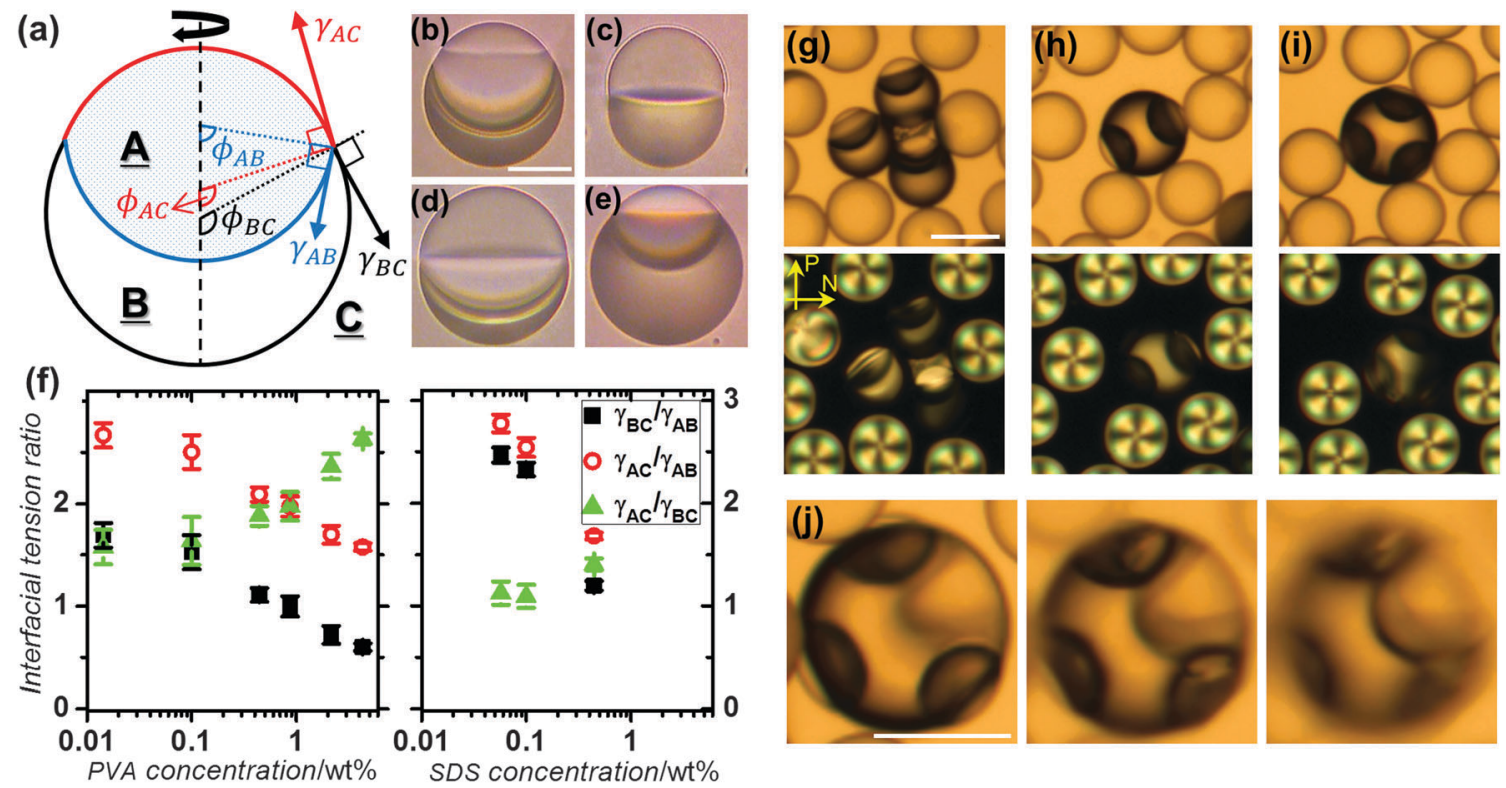

Fig. 2 Droplets with different morphologies. (a) Schematic of a Janus droplet with two compartments and three interfaces. Compartments $\underline{A}$, $\underline{B}$, and $\subseteq$ correspond to polymer, LC, and aqueous surfactant solution, respectively. Each colored arc indicates an interface. $\phi$ is defined as half the arc angle at the center of the arc; as an exception, $\phi_{\mathrm{AC}}$ is defined as being the supplement of the arc angle, i.e., $\pi$-arc angle. At the three-phase boundary, $\gamma$ denotes each interface's interfacial tension with a direction tangential to each arc. $\gamma_{A B}, \gamma_{B C}$, and $\gamma_{A C}$ are interfacial tensions at the polymer-LC, LC-water, and polymerwater interfaces, respectively. The rotating black arrow indicates the rotational symmetry of the droplet about the black dashed line. (b)-(e) Representative bright-field images of Janus droplets with different polymer : LC volume ratios at different concentrations of PVA/SDS in water. Note that these droplets are aligned by gravity and the side-view images were taken using a microscope turned on its side. Scale bar: $20 \mu \mathrm{m}$. The volume ratio between polymer and LC is (b and c) $1: 1$, (d) $3: 1$, and (e) $1: 3$. The type and concentration of surfactant is (b, d, e) PVA $0.1 \%$ (wt/vol) and (c) SDS $0.1 \%$ (wt/vol). (f) Experimentally determined ratios between interfacial tensions as a function of the concentration of surfactant (PVA or SDS) in water. Each data point is an average value derived from image analysis of more than five different droplets; the standard deviation of the sample is shown as an error bar. ( $g$-i) Brightfield (upper) and corresponding POM (lower) images of droplets with multiple compartments. Scale bar: $50 \mu \mathrm{m}$. The droplets in (g) are Janus droplets that consist of one polymer compartment and one LC compartment with the polymer:LC volume ratio set to be $1: 3$. The surrounding spherical droplets are similar Janus droplets viewed along the axis of rotational symmetry, as shown in Fig. $1 \mathrm{f}$ and $\mathrm{g}$. At the center of (h) and (i), we show droplets with two and three polymer compartments connected by a LC compartment, respectively. (j) Bright-field images of a droplet with four polymer compartments and a connecting LC compartment. Each image is in a different focal plane.

droplet and the three interfacial tensions. Each interfacial tension is a property of an interface where two different phases meet. The type and amount of surfactant molecules adsorbed at the interface affect the interfacial tension. In equilibrium, the three-phase boundary of any such droplet will adopt a unique configuration characterized by the relative angles between the three interfaces; this configuration requires that the vector sum of the three interfacial tensions equals 0 . The configuration is thus equivalent to a closed loop, where Neumann's triangle is formed by three vectors of interfacial tensions, $\gamma_{\mathrm{AB}}, \gamma_{\mathrm{BC}}$, and $\gamma_{\mathrm{AC}}$. In Fig. $2 \mathrm{a}, \gamma_{\mathrm{AB}}, \gamma_{\mathrm{BC}}$, and $\gamma_{\mathrm{AC}}$ are interfacial tensions at the polymer-LC, LC-water, and polymer-water interfaces, respectively. Note that we assume that the elasticity of the LC does not substantially affect the three-phase boundary, which is observed on a micrometer length scale. As a typical interfacial tension, $\gamma$, is of the order of $1 \mathrm{mN} \mathrm{m}^{-1}$ and a typical elastic modulus, $K$, of a $\mathrm{LC}$ is of the order of $10 \mathrm{pN}$, the interfacial energy dominates the LC elastic energy when the length scale, $L$, is greater than $\sim 10 \mathrm{~nm}$.

As shown in Fig. $2 \mathrm{~b}$ and c, changes in the type of surfactant used in the aqueous continuous phase lead to different Janus droplet morphologies. Interestingly, these different morphologies occur despite the fact that the sizes and volume ratios of the polymer and LC remain almost the same. Fig. $2 \mathrm{~b}$, d, and e also show another way of achieving different morphologies by tuning the polymer: LC volume ratio at a fixed surfactant concentration.

Analysis of the morphology of Janus droplets provides information about their interfacial tensions. In particular, by fitting their phase boundaries to arcs (see Experimental section), we can estimate the radii and arc angles that are associated with each phase boundary. From these data, it is possible to calculate the volume of each compartment (which was consistent with experimental expectations based on the sample preparation) and the various interfacial tension ratios. Construction of a Neumann triangle with three vectors only gives two independent forcebalance equations along two orthogonal directions; therefore, we can only calculate the relative ratios between the three interfacial tensions, i.e., not their absolute magnitudes. Fig. $2 \mathrm{f}$ shows how interfacial tension ratios change as a result of different concentrations of the two surfactants, poly(vinyl alcohol) (PVA) and sodium dodecyl sulfate (SDS). See also a theoretical morphology diagram in Fig. S1 of the ESI. $\dagger$ We expect (and assume) $\gamma_{\mathrm{AB}}$ to be constant regardless of surfactant conditions, because surfactant molecules in water are not adsorbed at the interface between 
polymer ( $\underline{\mathrm{A}})$ and $\mathrm{LC}(\underline{\mathrm{B}})$. The values of $\gamma_{\mathrm{BC}}$ and $\gamma_{\mathrm{AC}}$ decrease as the concentration of surfactant increases, because more surfactant is adsorbed at the corresponding interfaces. Note also that $\gamma_{\mathrm{AC}} / \gamma_{\mathrm{BC}}$ for SDS remains close to unity $\neq$ but is greater than 1.5 for PVA over the entire range of experimental surfactant concentrations. These findings are consistent with our observations of Janus droplets dispersed in the PVA solution (Fig. 2b); in this case, the interfacial area between the polymer compartment ( $\underline{\mathrm{A}})$ and the aqueous surfactant solution $(\underline{C})$ is much smaller than the interfacial area between the LC compartment ( $\underline{B})$ and the aqueous surfactant solution $(\underline{\mathrm{C}})$.

\section{Patchy colloids from coalescence of droplets}

In the process of carrying out these studies, we discovered that coalescence of LC Janus droplets provides a unique route to creating patchy colloidal particles/droplets. $\$$ Fig. 2g-j shows the optical microscopy images of LC droplets with multiple patches, i.e., multiple polymer compartments; the droplets are immersed in PVA solution. The droplets in Fig. $2 \mathrm{~g}$ are the same LC Janus droplets shown in Fig. 2e but if two of these Janus droplets merge by coalescence of their LC compartments then they evolve to become one LC droplet with two polymer droplet patches, as shown in Fig. 2h. Likewise, if three Janus droplets merge, the resulting droplet possesses three polymer droplet patches, as shown in Fig. 2i. The images suggest that the polymer droplets repel each other, perhaps because they distort the director field of the LC compartment; note that the LC directors at the polymer-LC interface are perpendicular to the interface. This repulsive interaction at the nematic interface explains the two-dimensional equiangular coordination of the patches shown in Fig. $2 \mathrm{~h}$ and i. Lastly, we made a LC droplet with four patches and three-dimensional coordination; the series of bright-field images in different focal planes in Fig. 2j indicates that three of the patches lie in the same plane, whereas the other patch is located out of the plane, similar to the vertices of a tetrahedron. The patchy colloids in sealed containers were stable for days; we never observed coalescence between patches.

Elucidation of the nature of the seemingly repulsive interactions between polymer droplets at the LC-water interface requires more investigation. The interactions and resulting pattern formation of colloids trapped at nematic interfaces

\footnotetext{
\# Our observation that $\gamma_{\mathrm{AC}} / \gamma_{\mathrm{BC}}$ is close to unity is consistent with previous research, ${ }^{40}$ although the latter work used a silicone oil that was much less viscous than ours, i.e., $13 \mathrm{cSt}$ versus $10000 \mathrm{cSt}$. Assuming that the high viscosity of our silicone oil does not affect $\gamma_{\mathrm{AC}}$, then $\gamma_{\mathrm{AC}}$ and $\gamma_{\mathrm{BC}}$ at the critical micelle concentration of SDS (approximately $0.23 \%(\mathrm{w} / \mathrm{w})$ ) are estimated to be 9.2 and $9.3 \mathrm{mN} \mathrm{m}^{-1}$, respectively. In addition, we can assume that $\gamma_{\mathrm{AC}}$ near a concentration of $0.5 \%$ (w/w) SDS is close to $9.0 \mathrm{mN} \mathrm{m}^{-1}$, because $\gamma$ changes little above the CMC. Consequently, we can estimate in Fig. $2 \mathrm{f}$ that $\gamma_{\mathrm{AB}}$, the interfacial tension between PDMS and $5 \mathrm{CB}$, is approximately $5 \mathrm{mN} \mathrm{m}^{-1}$. We readily calculate the other surface tensions with the assumption that $\gamma_{\mathrm{AB}}$ is constant regardless of the type and concentration of surfactants.

$\S$ Coalescence was promoted by mechanically agitating Janus emulsion droplets placed between the microscope slide and the cover glass. As a result, coalescence occurred randomly and patchy particles were concurrently produced with varying numbers of patches.
}

have been extensively debated. ${ }^{44-50}$ Depending on LC configurations that are determined by boundary conditions at LC-colloid, LC-water, or LC-air interfaces, several different patterns of colloidal assembly have been observed, e.g., closepacked hexagonal, chain-like or star-like patterns. A variety of interactions have been suggested as causing these effects: quadrupolar and dipolar elastic interactions, capillary interactions, strong reorganization of LC director fields at short distances, and even many-body interactions. Further systematic studies such as varying the size and elasticity of the LC compartment are needed to clarify these underlying interactions between polymer droplet patches.

\section{Tumbling and swimming LC Janus droplets}

The difference in density between the two compartments is perhaps the most notable anisotropic characteristic of LC Janus droplets. It leads to tumbling motion in response to gravity. As shown in Fig. 3a and b, LC Janus droplets float when their overall density is lower than the density of the dilute SDS background solution $\left(\rho_{\mathrm{w}-\mathrm{s}}, \sim 1.00 \mathrm{~g} \mathrm{~mL}^{-1}\right.$ at $\left.25{ }^{\circ} \mathrm{C}\right)$. The droplets also exhibit alignment along the direction of the gravitational field because the density of LC $\left(\rho_{\mathrm{LC}}, 1.01 \mathrm{~g} \mathrm{~mL} \mathrm{~m}^{-1}\right.$ at $25{ }^{\circ} \mathrm{C}$ ) is greater than $\rho_{\mathrm{w}-\mathrm{s}}$ and the density of polymer $\left(\rho_{\text {polymer }}, 0.97 \mathrm{~g} \mathrm{~mL} \mathrm{~m}^{-1}\right.$ at $\left.25{ }^{\circ} \mathrm{C}\right)$ is smaller than $\rho_{\mathrm{w}-\mathrm{s}}$. In particular, the center of mass of both compartments lies on the rotational axis because of rotational symmetry (see Fig. 2a). This rotational axis is aligned parallel to the direction of the gravitational field; the denser LC compartment is located at the bottom. If the direction of a LC Janus droplet deviates from the equilibrium alignment direction, the droplet experiences a restoring torque that causes it to align or tumble. Fig. 3c shows how a LC Janus droplet rotates after a sample was flipped over on a microscope stage. In Fig. 3c, the droplets in $0.5 \%$ (wt/vol) SDS solution relax to their equilibrium alignment directions within 10 seconds. If the concentration of the surfactant increases, the viscosity and the time needed for the droplets to recover their equilibrium alignment increase.

Finally, as the two compartments have different solubilities in aqueous surfactant solutions, it is possible to induce directional self-propelled motion of LC Janus droplets. It has been reported that LC droplets or oil droplets dispersed in certain types of highly concentrated aqueous surfactant solutions exhibit self-propelled motion. ${ }^{39,51}$ Whereas a full understanding of these phenomena is still evolving, the basic effect is believed to arise because of Marangoni stress. In particular, micelle-assisted solubilization of dispersed materials (e.g., LCs, oil emulsion droplets) into the continuous phase leads to vigorous transport of surfactant molecules among adsorbed layers and micelles and around droplets. Movement of dispersed droplets breaks the symmetry of transport phenomena and then a spatial gradient in the number of adsorbed surfactant molecules is generated on the surface of a droplet which in turn gives rise to Marangoni stress. This stress promotes surface flow at the interface, which couples with hydrodynamic flow inside and outside the droplet and causes the droplet to swim. ${ }^{39}$ 

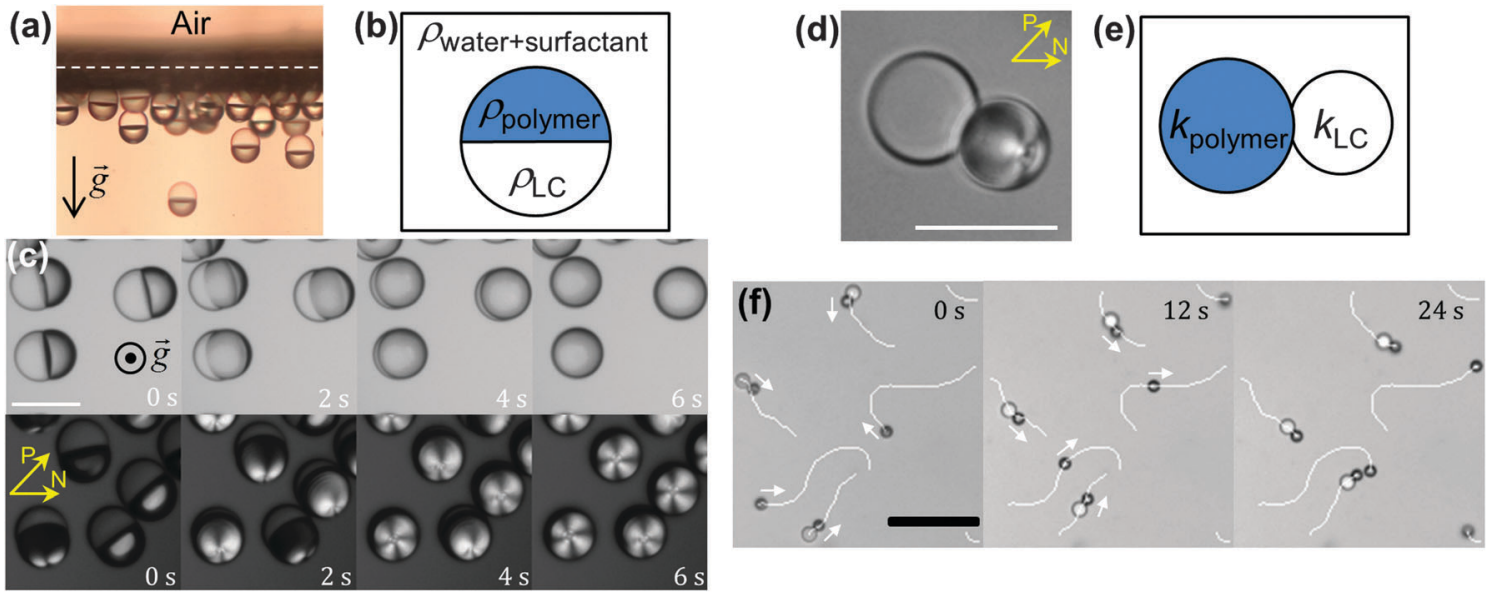

Fig. 3 Tumbling and swimming motion of Janus droplets. (a) Floating aligned Janus droplets near an air-water interface. The white dashed line indicates the air-water interface and the black arrow shows the direction of the gravitational field $(\vec{g})$. Herein, the lower compartment of the Janus droplets is liquid crystal (LC) and the upper one is polymer. (b) Schematic of a Janus droplet dispersed in an aqueous surfactant (PVA or SDS) solution with different densities $(\rho)$. (c) Tumbling of Janus droplets in response to gravity after flipping capillary containers containing the Janus droplets. Scale bar: $100 \mu$ m. Herein, the direction of the gravitational field $(\vec{g})$ points out of (perpendicular to) the plane of the image. The upper and lower rows show bright-field and POM images at intervals of 2 seconds, respectively; single-headed yellow arrows indicate the directions of the pass axes of the polarizer (P) and analyzer (N). In POM images, birefringent LC compartments appear bright. (d) POM image of a Janus droplet dispersed in $10 \%$ (wt/vol) $\mathrm{C}_{14} \mathrm{TAB}$ solution. The birefringent LC compartment on the right-hand side displays a brightness pattern because of the polarizer (P) and analyzer (N). Scale bar: $20 \mu \mathrm{m}$. (e) Schematic of a Janus droplet with different solubilities $(k)$ in $C_{14} T A B$ solution. (f) Self-propelled motion of Janus droplets and LC droplets. Scale bar: $100 \mu \mathrm{m}$. Bright-field images are shown at intervals of 12 seconds. The overlaid white solid lines and white arrows indicate the droplet trajectories and directions of motion, respectively; the snowman-like droplets with two compartments are Janus droplets; the spherical droplets are LC droplets without a polymer compartment. In the case of Janus droplets, the LC compartment, which has a thicker boundary than the polymer compartment, leads the self-propelled motion as a 'truck', which pulls the polymer compartment as a 'trailer'.

We placed LC Janus droplets into the same class of concentrated surfactant solution, i.e., $\sim 10 \%(\mathrm{wt} / \mathrm{vol})$ tetradecyltrimethylammonium bromide $\left(\mathrm{C}_{14} \mathrm{TAB}\right)$ solution. In this case, the LC compartment slowly dissolved into the background phase, ${ }^{39,51}$ whereas the polymer compartment did not dissolve. It has been reported $^{51}$ that the radius, $r$, of a spherical LC droplet decreases linearly with time in aqueous $\mathrm{C}_{14} \mathrm{TAB}$ surfactant solution and the solubilization rate, $-\mathrm{d} r / \mathrm{d} t$, increases non-linearly but monotonically with increasing surfactant concentration; the measured solubilization rate in $\sim 10 \%(\mathrm{wt} / \mathrm{vol}) \mathrm{C}_{14} \mathrm{TAB}$ solution is approximately $5 \mathrm{~nm} \mathrm{~s}^{-1}$. ${ }^{1}$ Therefore, the LC compartment shrinks and eventually disappears whereas the volume of the polymer compartment remains constant. In Fig. 3d, we show a LC Janus droplet in this concentrated $\mathrm{C}_{14} \mathrm{TAB}$ solution; it displays a snowman-like morphology. This morphology arises because the high concentration of surfactant in the background phase induces very low interfacial tensions at the LC-water $\left(\gamma_{\mathrm{BC}}\right)$ and polymer-water $\left(\gamma_{\mathrm{AC}}\right)$ interfaces relative to the tension at the LC-polymer interface $\left(\gamma_{\mathrm{AB}}\right)$. (For further clarification, see also a theoretical morphology diagram in Fig. S1 of the ESI. $\dagger$ ) In Fig. 3d, the brightness pattern in the LC compartment (right) results from the birefringence of the LC; the pass axes of the crossed polarizers are shown as single-headed arrows in the figure. Note that the off-center cross-like pattern with an off-center radial point defect indicates a radial LC director configuration with perpendicular anchoring at the LC-water interface imposed by the surfactant. Moreover, note that Marangoni stress-induced flow inside the LC compartment causes the position of the point defect to deviate from the center of the droplet. ${ }^{51}$
These LC Janus droplets exhibit self-propelled motion in the surfactant solution at a speed of $\sim 10 \mu \mathrm{m} \mathrm{s}^{-1}$. In Fig. 3f, both spherical LC droplets and snowman-like LC Janus droplets (with two compartments) display self-propelled motion (see Movie S1 in the ESI $\dagger$ ). Spherical LC droplets swim approximately twice as fast as snowman-like LC Janus droplets. On the other hand, non-dissolving polymer droplets do not exhibit self-propelled motion; small polymer droplets of a few microns in diameter exhibit Brownian motion only in the same surfactant solution. These observations suggest that the LC Janus droplet is propelled by the LC compartment only, with the polymer compartment that is attached to the LC compartment acting like a trailer attached to a truck. Furthermore, in contrast to spherical droplets, the self-propelled motion of LC Janus droplets is always directional; the "truck-like" LC compartment leads the motion of the LC Janus droplet. We presume that the swimming direction is determined by hydrodynamics around the swimming Janus droplet; namely, Marangoni stress-induced flow around the LC compartment is compatible with the polymer compartment attached to the rear side of the swimming Janus droplet. Ultimately, this directional motion may facilitate control over colloid hydrodynamics and provide a means of cargo delivery via design of the shape and size of the swimming droplet. In a similar way, we can preset the lifetime of the swimming droplet; eventually the shrinking LC compartment becomes too small to propel the attached polymer compartment. In the end, the LC compartment disappears and the inactive polymer compartment remains (see Movie S2 in the ESI†). 


\section{Conclusions}

We introduced and prepared LC Janus droplets, which consist of two compartments: one polymer and one LC. The synthesis scheme employs solvent-induced phase separation in emulsion droplets generated by microfluidics. We explored how the surfactant concentration of the background phase, the volume ratios of the two compartments, and the coalescence of multiple Janus droplets lead to various droplet morphologies. Besides demonstrating the preparation method, we investigated the effects of three different anisotropic characteristics of Janus droplets that arise from the unique combination of a polymer and a LC. Firstly, while the polymer compartment is optically isotropic, the LC compartment is optically birefringent and the alignment structure of the LC molecules that produces this birefringence can be controlled via both the surface anchoring conditions of the LC and the confining geometry of the LC compartment. In addition, differences in density between the two compartments and the aqueous background phase produce gravity-induced alignment and tumbling of Janus droplets. Lastly, LC Janus droplets exhibit directional self-propelled motion in a concentrated surfactant solution because of differential solubility within the surfactant solution and shape anisotropy; the LC compartment always leads the swimming process.

Further study of LC Janus droplets could produce new applications of Janus colloids. The LC compartment provides a new set of "knobs" for control based on LC director structures that can be configured (and reconfigured) by external fields or boundary conditions. For example, the LC director configuration and resulting optical appearance of the LC compartments under polarizers or wave plates can change due to adsorption of amphiphilic molecules at the LC-water interface, while the other compartment is unaffected. Interestingly, compared to spherical LC droplets, the "sensitivity" of LC Janus droplets to adsorbing molecules can be tuned via the non-LC compartment; the energetics of an adsorbate-triggered transition, for example, is affected by the size and shape of the LC compartment as well as the anchoring conditions at the interface between two compartments of Janus droplets. Furthermore, the LC materials used in Janus droplets are not limited to the nematic phase; the LC compartment can comprise a chiral nematic, smectic, or any other liquid crystalline phase as long as they are not fully miscible with the polymer or background phase. This variety of LC phases can endow Janus droplets with other unique features such as periodic indices of refraction (associated with chiral nematic phases), which, for example, have implications for photonics. In addition, the preparation schemes offer the possibility of the creation of new LC compartments with non-trivial geometries and boundary conditions.

Finally, the interesting dynamical behavior of LC Janus droplets opens up new possibilities for Janus colloids in soft matter. From the aligning and tumbling of Janus droplets, we can derive information about the densities of components or the rheology of a background phase. The birefringence and resulting optical patterns of the LC compartment, in particular, facilitate easy observation of rotation effects. Furthermore, swimming LC
Janus droplets with multiple compartments can be utilized to transport external bodies from one place to another and to study the hydrodynamics of non-spherical swimming droplets. For example, one or more polymer compartments, which are inactive and do not dissolve, can contain nanoparticles or compatible chemicals while the LC compartment drives the motion of the whole droplet until it is completely dissolved in the background phase. Furthermore, as shown in Fig. 3d, the shape of the swimming droplet is close to being elongated, in contrast to common spherical model swimming particles. The volume of the inactive tail compartment and even their number, e.g., the multiple polymer patches in Fig. $2 \mathrm{~h}-\mathrm{j}$, can be controlled to have different hydrodynamics. Consequently, the LC Janus droplet has the potential to be an excellent model system for the study of the collective behavior of swimming particles mediated by tunable hydrodynamic interactions.

\section{Acknowledgements}

Authors thank Zoey S. Davidson for helpful discussions and gratefully acknowledge financial support from the National Science Foundation through DMR-1205463 and DMR-1120901, and support from the National Aeronautics and Space Administration (NASA) through NNX08AO0G. J.J. also acknowledges support from the Korean Government through IBS-R020-D1.

\section{Notes and references}

1 J. Du and R. K. O'Reilly, Chem. Soc. Rev., 2011, 40, 2402-2416.

2 J. Hu, S. Zhou, Y. Sun, X. Fang and L. Wu, Chem. Soc. Rev., 2012, 41, 4356-4378.

3 Q. Chen, S. C. Bae and S. Granick, Nature, 2011, 469, 381-384.

4 S. Gangwal, O. J. Cayre, M. Z. Bazant and O. D. Velev, Phys. Rev. Lett., 2008, 100, 1-4.

5 M. Lattuada and T. A. Hatton, Nano Today, 2011, 6, 286-308.

6 N. Zhao and M. Gao, Adv. Mater., 2009, 21, 184-187.

7 C.-H. Chen, A. R. Abate, D. Lee, E. M. Terentjev and D. a. Weitz, Adv. Mater., 2009, 21, 3201-3204.

8 S. K. Smoukov, S. Gangwal, M. Marquez and O. D. Velev, Soft Matter, 2009, 5, 1285-1292.

9 S.-H. Kim, J. Y. Sim, J.-M. Lim and S.-M. Yang, Angew. Chem., Int. Ed., 2010, 49, 3786-3790.

10 S. Jiang, Q. Chen, M. Tripathy, E. Luijten, K. S. Schweizer and S. Granick, Adv. Mater., 2010, 22, 1060-1071.

11 I. Buttinoni, J. Bialké, F. Kümmel, H. Löwen, C. Bechinger and T. Speck, Phys. Rev. Lett., 2013, 110, 1-5.

12 J. Palacci, S. Sacanna, A. P. Steinberg, D. J. Pine and P. M. Chaikin, Science, 2013, 339, 936-940.

13 H. Takei and N. Shimizu, Langmuir, 1997, 13, 1865-1868.

14 J. Ge, Y. Hu, T. Zhang and Y. Yin, J. Am. Chem. Soc., 2007, 129, 8974-8975.

15 R. K. Shah, J.-W. Kim and D. A. Weitz, Adv. Mater., 2009, 21, 1949-1953. 
16 A. Kumar, B. J. Park, F. Tu and D. Lee, Soft Matter, 2013, 9, 6604 .

17 T. Nisisako, T. Torii, T. Takahashi and Y. Takizawa, Adv. Mater., 2006, 18, 1152-1156.

18 S.-N. Yin, C.-F. Wang, Z.-Y. Yu, J. Wang, S.-S. Liu and S. Chen, Adv. Mater., 2011, 23, 2915-2919.

19 O. Cayre, V. N. Paunov and O. D. Velev, J. Mater. Chem., 2003, 13, 2445-2450.

20 Y. Lu, H. Xiong, X. Jiang, Y. Xia, M. Prentiss and G. M. Whitesides, J. Am. Chem. Soc., 2003, 125, 12724-12725.

21 M. Conradi, M. Ravnik, M. Bele, M. Zorko, S. Žumer and I. Muevič, Soft Matter, 2009, 5, 3905-3912.

22 H. R. Sheu, M. S. El-Aasser and J. W. Vanderhoff, J. Polym. Sci., Part A: Polym. Chem., 1990, 28, 629-651.

23 J.-W. Kim, R. J. Larsen and D. A. Weitz, J. Am. Chem. Soc., 2006, 128, 14374-14377.

24 F. Tu and D. Lee, J. Am. Chem. Soc., 2014, 136, 9999-10006.

25 H. Gu, Z. Yang, J. Gao, C. K. Chang and B. Xu, J. Am. Chem. Soc., 2005, 127, 34-35.

26 L. Hong, S. Jiang and S. Granick, Langmuir, 2006, 22, 9495-9499.

27 K.-H. Roh, D. C. Martin and J. Lahann, Nat. Mater., 2005, 4, 759-763.

28 A. X. Lu, K. Jiang, D. L. Devoe and S. R. Raghavan, Langmuir, 2013, 29, 13624-13629.

29 C.-H. Choi, S.-M. Kang, S. H. Jin, H. Yi and C.-S. Lee, Langmuir, 2015, 31, 1328-1335.

30 J. Yoon, K. J. Lee and J. Lahann, J. Mater. Chem., 2011, 21, 8502.

31 N. Saito, Y. Kagari and M. Okubo, Langmuir, 2006, 22, 9397-9402.

32 T. Higuchi, A. Tajima, H. Yabu and M. Shimomura, Soft Matter, 2008, 4, 1302-1305.

33 L. D. Zarzar, V. Sresht, E. M. Sletten, J. a. Kalow, D. Blankschtein and T. M. Swager, Nature, 2015, 518, 520-524.

34 N. G. Min, B. Kim, T. Y. Lee, D. Kim, D. C. Lee and S.-H. Kim, Langmuir, 2015, 31, 937-943.
35 J. Jeong, E. Um, J. K. Park and M. W. Kim, RSC Adv., 2013, 3, 11801-11806.

36 A. Fernández-Nieves, G. Cristobal, V. Garcés-Chávez, G. C. Spalding, K. Dholakia and D. a. Weitz, Adv. Mater., 2005, 17, 680-684.

37 O. O. Prishchepa, A. V. Shabanov and V. Y. Zyryanov, Phys. Rev. E: Stat., Nonlinear, Soft Matter Phys., 2005, 72, 031712.

38 A. Fernández-Nieves, Soft Matter, 2006, 2, 105-108.

39 S. Herminghaus, C. C. Maass, C. Krüger, S. Thutupalli, L. Goehring and C. Bahr, Soft Matter, 2014, 10, 7008-7022.

40 P. B. Umbanhowar, V. Prasad and D. A. Weitz, Langmuir, 2000, 16, 347-351.

41 N. Gogibus, U. Maschke, F. Benmouna, B. Ewen, X. Coqueret and M. Benmouna, J. Polym. Sci., Part B: Polym. Phys., 2001, 39, 581-588.

42 P. Drzaic, Liquid crystal dispersions, World Scientific, Singapore, 1995.

43 P. G. De Gennes and J. Prost, The Physics of Liquid Crystals, Clarendon Press, Oxford, 2nd edn, 1993.

44 X. Wang, D. S. Miller, J. J. de Pablo and N. L. Abbott, Adv. Funct. Mater., 2014, 24, 6219-6226.

45 I. I. Smalyukh, S. Chernyshuk, B. I. Lev, A. B. Nych, U. Ognysta, V. G. Nazarenko and O. D. Lavrentovich, Phys. Rev. Lett., 2004, 93, 117801.

46 M. A. Gharbi, M. Nobili, M. In, G. Prévot, P. Galatola, J.-B. Fournier and C. Blanc, Soft Matter, 2011, 7, 1467-1471.

47 M. A. Gharbi, M. Nobili and C. Blanc, J. Colloid Interface Sci., 2014, 417, 250-255.

48 G. M. Koenig, I.-H. Lin and N. L. Abbott, Proc. Natl. Acad. Sci. U. S. A., 2010, 107, 3998-4003.

49 M. Oettel, a. DomInguez, M. Tasinkevych and S. Dietrich, Eur. Phys. J. E: Soft Matter Biol. Phys., 2009, 28, 99-111.

50 V. Pergamenshchik, Phys. Rev. E: Stat., Nonlinear, Soft Matter Phys., 2009, 79, 011407.

51 K. Peddireddy, P. Kumar, S. Thutupalli, S. Herminghaus and C. Bahr, Langmuir, 2012, 28, 12426-12431. 